\title{
Mental Illness Stigma in Mental Health Professionals
}

\author{
A.J.Marques, J.Figueiras, C.Queiros \\ ${ }^{1}$ Psychosocial Rehabilitation Laboratory, Faculty of Psychology and Educational Sciences, Porto \\ University and School of Allied Health Sciences, Porto Polytechnic Institute, ${ }^{2}$ Associação de \\ Familiares e Utentes do Hospital de Magalhães Lemos, ${ }^{3}$ Psychosocial Rehabilitation Laboratory, \\ FPCEUP/ESTSP, Porto, Portugal
}

Introduction: Mental illness is still associated with myths, prejudices and stereotypes, despite the growing investment in research and treatment in this area. The stigma still one of the major obstacle to recovery of persons with severe mental illnesses. Stigma affects an individual's self-esteem, increases dysfunction, and poses problems to patients with regard to housing and employment. Stigma is a culturally induced barrier to recovery. Although studies have examined experiences of stigma by users of mental health services and stigmatizing attitudes in the general public, little attention has been paid to the experiences and attitudes of mental health professionals.

Aims: To explore and clarify the presence or absence of stigmatizing attitudes in mental health professionals.

Methods: 24 qualitative interviews to mental health professionals working at psychiatric services at a general hospital, a psychiatric specialized hospital, and a Community Association in Oporto, Portugal.

Results: The analysis of the discursive material provided by social workers, nurses, psychiatrists, psychologists and occupational therapists reveals the presence of stigmatizing beliefs and attitudes regarding mental illness, in spite of the education or place they work. There are a few exceptions, like age and occupation that seems to influence the professionals' perspectives.

Conclusions: These results might denote that changes in professionals' attitudes are primarily a result of their personal experiences in and out their professional environment. Therefore, it is important to create programs focused on these issues, to promote training, multidisciplinary discussion and reflection allowing a greater awareness of stigmatizing attitudes and beliefs in order to minimize them. 